\title{
New tool for the diagnosis of enzootic bovine leukosis virus infection
}

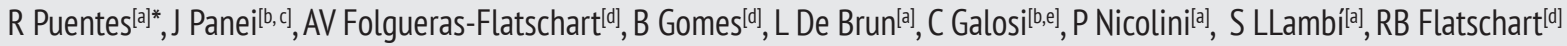

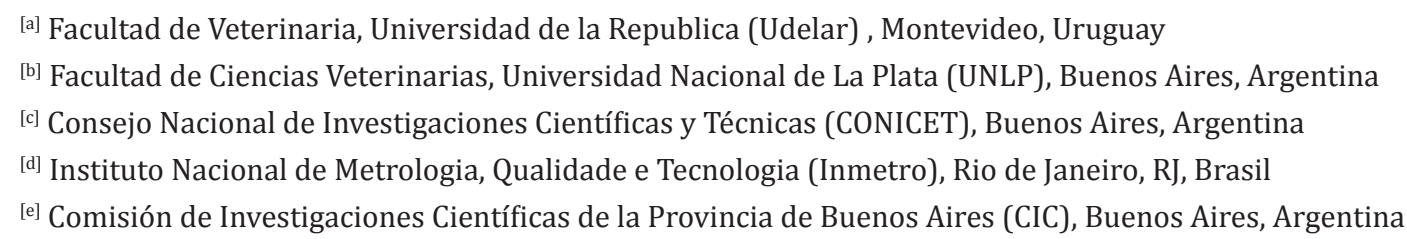

${ }^{*}$ Corresponding author

e-mail: rpuentes@adinet.com.uy

\section{Abstract}

Droplet digital PCR (ddPCR) is a highly sensitive tool developed for the detection and quantification of nucleic acids. It can detect and quantify small percentage differences of rare variants, presenting significant advantages over existing tools. This paper describes for the first time the use of the ddPCR for detecting the presence of DNA (provirus) of the Bovine leukemia virus (BLV). Sixty seven blood samples from animals naturally exposed to the virus were analyzed and the results were compared with those obtained by nested PCR (nPCR). The 62.7\% (42/67) of the samples was positive by nPCR, while $89.5 \%$ (60/67) were positive by ddPCR. The mean and median number of proviral copies samples that were positive by ddPCR and negative by nPCR was 23 and 21 proviral copies/uL of genomic DNA, respectively. For positive values by both techniques the mean and median number of proviral copies samples were 2479 and 554 proviral/uL copies of genomic DNA respectively. This shows that the negative samples by nPCR even contain considerably low number of copies of provirus that are not being detected. In conclusion, the ddPCR turned out to be a promising tool for the detection and absolute quantification of BLV in peripheral blood of cattle. 